Meta

Journal des tradlucteurs

Translators' Journal

\title{
Esquisse d'une méthode de traduction au dictaphone
}

\section{Jean-Marc Gouanvic}

Volume 21, numéro 4, décembre 1976

URI : https://id.erudit.org/iderudit/002951ar

DOI : https://doi.org/10.7202/002951ar

Aller au sommaire du numéro

Éditeur(s)

Les Presses de l'Université de Montréal

ISSN

0026-0452 (imprimé)

1492-1421 (numérique)

Découvrir la revue

Citer cet article

Gouanvic, J.-M. (1976). Esquisse d'une méthode de traduction au dictaphone. Meta, 21(4), 252-255. https://doi.org/10.7202/002951ar d'utilisation que vous pouvez consulter en ligne.

https://apropos.erudit.org/fr/usagers/politique-dutilisation/ 


\section{Esquisse d'une méthode de traduction au dictaphone}

Le présent article a pour but de familiariser l'utilisateur du dictaphone avec son fonctionnement et de présenter l'une des méthodes empiriques possibles ainsi que quelques conseils qui, nous l'espérons, seront utiles aux étudiants.

La traduction au dictaphone nécessite un examen attentif des mécanismes qu'elle met en œuvre, certains de ces derniers différant sensiblement de ceux qu'implique l'emploi d'une machine à écrire. Il en résulte tout naturellement une méthodologie que nous examinerons plus bas.

\section{Similitude entre les deux procédés}

Une similitude importante existe entre les deux procédés. Ceux qui se servent d'une machine à écrire procèdent souvent selon une ou plusieurs méthodes qu'ils ont adaptées à leur tempérament et à leur formation. (On est plus ou moins rapide, plus ou moins au courant du sujet à traduire.) Peu à peu, cependant, s'impose à tous la traduction directe, c'est-à-dire sans brouillon. Entre le texte de la langue de départ et celui de la langue d'arrivée, il est important, pour que l'efficacité soit optimale, de réduire les intermédiaires. Cette remarque vaut pour l'emploi du dictaphone plus encore que pour celui de la machine à écrire. Quels que soient les appareils utilisés, il est essentiel d'effectuer une recherche très soigneuse des termes, expressions, etc. inconnus, ou des particularités du texte (y compris des passages obscurs), avant la phase finale de la rédaction ou de la dictée. On voit donc que, à ce stade, le processus est le même. Pour que l'emploi du dictaphone atteigne une efficacité maximale, il convient d'être averti de certaines servitudes inhérentes à la dictée.

\section{Servitudes propres au dictaphone}

Avec la machine à écrire, il semble possible, lorsque la traduction juste n'apparaît pas immédiatement, de laisser des blancs, ou de se contenter, " pour l'instant ", d'une formule approximative. Avec le dictaphone cela n'est pas praticable. Pour des raisons d'ordre essentiellement technique, il n'est pas conseillé de laisser des blancs sur la bande, le texte finalement choisi pouvant par exemple dépasser la longueur prévue. 
Il est en outre dangereux de multiplier les incertitudes et les plages d'indécision; par manque d'efficacité, le travail final risque d'être médiocre, décousu. Une préparation très soigneuse du texte doit permettre de pallier ces inconvénients.

\section{Préparation du texte à traduire}

En quoi consiste la préparation? Il importe de distinguer deux phases préliminaires essentielles qui correspondent à une première et à une deuxième lecture du texte.

\section{Première lecture}

a) Dès la première lecture, il convient de noter directement sur le texte à traduire tout ce qui vient à l'esprit du traducteur : tournures, inversions propres au français, termes particuliers.

Il est essentiel de ne rien perdre de la première impression de lecture.

b) Au cours de la première lecture également, il faut souligner les points obscurs ou mal saisis du texte.

\section{Deuxième lecture}

Se munir de fiches et y apporter les termes et expressions qui font difficulté. Cet aspect de la méthode est important, en particulier pour l'uniformité de la terminologie. Il est courant en effet que, pour une expression ou un terme, trois traductions soient acceptables. Tout au long du texte, il importe (sauf exception) de s'en tenir à la même formule pour que le lecteur puisse la reconnaître sans difficulté.

$N . B$. : Il est clair que le texte doit être lu et examiné plus de deux fois; nous n'abordons ici que les deux premières lectures, souvent négligées alors qu'elles sont fondamentales pour réaliser un travail efficace et de bonne qualité.

Quand tout le texte a été examiné à la loupe, le moment est venu de passer à la recherche, à la consultation du fichier, du dictionnaire, fiches en main (cf. deuxième lecture ci-dessus). Lorsque les fiches existent au fichier central de l'entreprise, on se borne à les recopier, en choisissant, si un choix est possible, une formule pour la cohésion du vocabulaire du texte. Quel que soit l'appareillage utilisé, la recherche doit être systématique. Nous n'insisterons donc pas davantage sur cet aspect.

La recherche est maintenant terminée. Le traducteur dispose de toutes les données. Entre le moment où le texte à traduire a été reçu et la fin de la recherche, pour 3000 mots environ d'un texte de difficulté moyenne, il a pu s'écouler une demi-journée de travail de recherche, de lecture et de compréhension. Le traducteur n'a pas encore touché à son dictaphone. Le moment est venu de passer à la dictée. 


\section{Le dictaphone - aspect technique}

Un dictaphone est un simple magnétophone à triple commandes: les premières sont celles d'un magnétophone ordinaire, à chaque touche correspondant une fonction (défilement arrière, défilement avant, enregistrement, écoute, etc.). Les deuxièmes commandes sont celles qui intéressent le traducteur : en effet, en une seule main et en un seul bouton à triple position, il dispose des commandes d'enregistrement, de défilement arrière et d'écoute de la bande. (Le traducteur n'a donc à s'occuper que de ces commandes pour enregistrer.) Les troisièmes commandes sont les commandes à pied qui ne servent qu'au dactylographe.

\section{Que dicter?}

Il devrait, en principe, être possible de déterminer de façon précise ce qui doit être dicté. En réalité, ce qu'il faut dicter dépend à la fois de l'entreprise pour laquelle on travaille et du dactylographe chargé de transcrire le texte. En règle générale, il faut dicter tout ce qui n'est pas absolument évident dans un texte; entre autres: la ponctuation, les majuscules et les minuscules insolites, les pluriels et les singuliers, les accords peu évidents (car le dactylographe ne peut pas suivre le texte, le taper et en comprendre le sens tout à la fois, surtout lorsque le sujet lui est inconnu).

En résumé, il faut donc dicter et épeler tout ce qui peut paraître peu usité et peu clair.

\section{6. À quel rythme dicter?}

Le débit est, semble-t-il, une question personnelle, chacun dictant au rythme qui lui convient. Il est cependant préférable de ne pas adopter un rythme trop rapide, car il faut bien articuler. On évite ainsi au dactylographe de revenir souvent en arrière, et de s'évertuer à comprendre un mot prononcé indistinctement. Le travail définitif est d'autant meilleur que le rythme est égal et sans-à-coups. Si le travail préliminaire est bien fait, la dictée ne doit pas présenter trop de difficultés, du point de vue du traducteur. Elle n'est qu'affaire d'habitude.

N.B. : On se rapportera avec profit à la directive générale du service de traduction (section française) de l'O.N.U. relative à l'emploi du magnétophone (réf. : SFTR/1, avril 1974, p. 181-182).

\section{Considérations générales}

a) $Y$ a-t-il des textes qui ne se prêtent pas à la dictée?

Selon nous, tout texte peut être dicté. Il serait trop long de passer en revue tous les genres de traductions demandées aux traducteurs. L'expérience montre que 1a dictée n'est que question de méthode et de préparation.

\section{b) Le travail est-il moins bon qu'à la machine à écrire?}

$\mathrm{Au}$ début, il peut l'être; mais les mécanismes de la dictée s'acquièrent assez vite et, à terme, permettent de réaliser un travail de bonne qualité. Une traduction faite au dictaphone se dégage plus facilement de l'original et gagne donc en 
vivacité. La parole est une épreuve : il faut prononcer une phrase pour en éprouver la cohérence, l'élégance, l'équilibre. (On sait que certains écrivains n'écrivaient jamais que ce qu'ils avaient dit au préalable à haute voix.)

Il est en outre certain qu'un texte se bâcle aussi facilement à la machine à écrire qu'à la machine à dicter.

L'antagonisme qu'on a bien voulu voir entre production et perfection, quantité et qualité, risque d'être faux. Certaines personnes se sentent inhibées devant une machine à écrire, d'autres devant une machine à dicter. Les jeunes traducteurs n'ayant pas acquis les réflexes de la traduction à la machine à écrire ont tout intérêt à s'essayer au dictaphone. La machine à écrire peut les stimuler considérablement.

\section{Examen du texte dactylographié}

Toutefois, si le texte a des faiblesses (il est peu probable qu'il n'en contienne pas), il est toujours loisible au traducteur de le retoucher, quand il l'a reçu de la dactylographie et avant de le soumettre à la révision. En réalité, le traducteur a intérêt à relire son texte très soigneusement. Une traduction faite dans les conditions professionnelles courantes a peu de chances d'être parfaite; aussi ne faut-il rien laisser passer de ce que l'on peut remarquer et corriger.

Quant à la confrontation du texte traduit avec l'original, le réviseur peut souhaiter s'en charger lui-même ou le demander au traducteur. C'est la charge de travail de l'un et de l'autre qui pourra en décider.

\section{Conclusion}

En conclusion et à titre d'exemple, il nous est apparu qu'un texte de 3000 mots et de difficulté moyenne exige une demi-journée de préparation pour environ une journée de dictée et de relecture. 